\title{
Pion interferometry for hydrodynamical expanding source with a finite baryon density
}

\author{
W. N. Zhang ${ }^{1,2}$, M. J. Efaaf ${ }^{1}$, Cheuk-Yin Wong $^{2,3}$, and M. Khaliliasr ${ }^{1}$ \\ ${ }^{1}$ Department of Physics, Harbin Institute of Technology, Harbin, 150006, P. R. China \\ ${ }^{2}$ Physics Division, Oak Ridge National Laboratory, Oak Ridge, TN 37831, U.S.A. \\ ${ }^{3}$ Department of Physics, University of Tennessee, Knoxville, TN 37996, U.S.A.
}

(Dated: September 17, 2018)

\begin{abstract}
We calculate the two-pion correlation function for an expanding hadron source with a finite baryon density. The space-time evolution of the source is described by relativistic hydrodynamics and the HBT radius is extracted after effects of collective expansion and multiple scattering on the HBT interferometry have been taken into account, using quantum probability amplitudes in a path-integral formalism. We find that this radius is substantially smaller than the HBT radius extracted from the freeze-out configuration.

PACS numbers: 25.75.-q, 25.75.Gz
\end{abstract}

The Bose-Einstein correlation of identical bosons produced in high-energy heavy-ion collisions, also known as the HBT effect, is an important tool for the study of the space-time structure of the emitting source [1]. As the source expands, cools, and freezes out, it is important to know what source distribution the HBT interferometry measures. Is it the freeze-out source distribution, the initial source distribution, or the initial source distribution modified by absorption and expansion? The conventional viewpoint is that the HBT interferometry measures the freeze-out configuration because rescattering of source particles are assumed to lead to a chaotic source. However, the extracted experimental HBT radii are insensitive to the collision energy, and $R_{\text {out }} / R_{\text {side }} \approx 0.9-1.1$ at RHIC 2, 3]. These general features cannot be understood within the conventional viewpoint $[4,5,6]$.

The validity of the conventional assumption on HBT is recently questioned as it was pointed out that because HBT is purely a quantum-mechanical phenomenon, the effects of rescattering and collective dynamics must be investigated within a quantum-mechanical context 7]. As fully quantum treatment of heavy-ion collisions is beyond the scope of our present investigation, we shall describe the gross dynamics of the hadron system with a finite baryon density by classical hydrodynamics. In this letter, we follow Wong's work 7] and take into account the effects of collective expansion and multiple scattering by constructing the quantum probability amplitude using Glauber's multiple scattering model and the trajectories of test particles in the path-integral framework.

The hydrodynamics and the composition of the fluid depend on the collision energy. We study in this work the dynamics of heavy-ion collisions in the energy range corresponding to about $10 \mathrm{GeV}$ per nucleon beam energy in fixed target collisions in which nucleons and deltas play an important role and pions are produced dominantly from deltas and absorbed by nucleons.

Relativistic hydrodynamics has been extensively applied to high-energy heavy-ion collisions [ 8$]$. The energy momentum tensor of a thermalized fluid cell in the centerof-mass frame is [8]

$$
T^{\mu \nu}(x)=[\epsilon(x)+p(x)] u^{\mu}(x) u^{\nu}(x)-p(x) g^{\mu \nu},
$$

where $x$ is the space-time coordinate, $\epsilon, p$, and $u^{\mu}=$ $\gamma(1, \boldsymbol{v})$ are respectively the energy density, pressure, and 4 -velocity of the cell, and $g^{\mu \nu}$ is the metric tensor. The local conservation of energy and momentum can be expressed by

$$
\partial_{\mu} T^{\mu \nu}(x)=0, \quad(\nu=0,1,2,3) .
$$

The conservation of baryon number gives

$$
\partial_{\mu} j^{\mu}(x)=0,
$$

where $j^{\mu}=n_{b}(x) u^{\mu}$ is the four-current-density of baryon ( $n_{b}$ is baryon density). In the thermalized cell, the density number $n_{i}$ of the particle species $i$, the energy density $\epsilon_{i}$, the pressure $p_{i}$, and the local velocity of sound $c_{s}$ can be obtained as a function of local temperature $T(x)$ and local chemical potential $\mu_{i}(x)$ by assuming an ideal hadron gas. For simplicity, we consider a hadronic gas consisting of nucleons, $\Delta(1232)$, and pions with spherical geometry as in Ref. [9]. For spherical geometry Eqs. (2) and (3) become [9]

$$
\begin{aligned}
\partial_{t} E+\partial_{r}[(E+p) v] & =-F, \\
\partial_{t} M+\partial_{r}(M v+p) & =-G, \\
\partial_{t} N+\partial_{r}(N v) & =-U,
\end{aligned}
$$

where $E \equiv T^{00}, M \equiv T^{o r}, N \equiv n_{b} \gamma$,

$$
F=\frac{2 v}{r}(E+p), \quad G=\frac{2 v}{r} M, \quad U=\frac{2 v}{r} N .
$$

In order to bring out the salient features of the effects under consideration, we shall carry out model calculations for some idealized space-time configurations. In these model calculations, we assume that the initial velocity $v(0, r)=0$, and the initial energy density is a Gaussian distribution $\epsilon(0, r)=\epsilon_{0} e^{-r^{2} / 2 R_{0}^{2}}$ [10]. In a local equilibrium cell, we have $\mu_{\pi}=0, \mu_{N}=\mu_{\Delta} \equiv$ $\mu_{B}$. Using the HLLE scheme [9] and with the relations of $\epsilon\left(T(x), \mu_{B}(x)\right), p\left(T(x), \mu_{B}(x)\right), n_{b}\left(T(x), \mu_{B}(x)\right)$, and $c_{s}\left(T(x), \mu_{B}(x)\right)$, we can get the solution of the hydrodynamical equations for $F=G=U=0$. Then, using the 


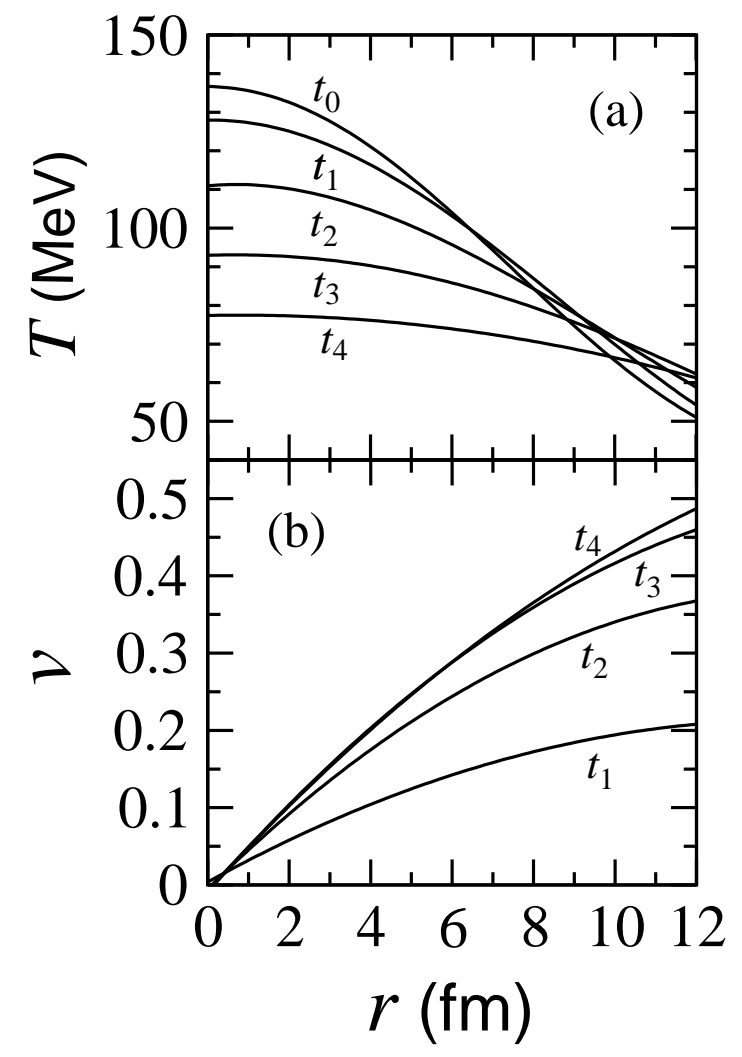

FIG. 1: Temperature (a) and velocity (b) profiles for the expanding source at $t_{n}=3 n \mathrm{fm} / \mathrm{c}$.

Sod's operator splitting method [9], we can obtain the solution for Eqs. (4), (5), and (6).

Figure 1 shows the temperature and velocity for a spherical expanding source with $\epsilon_{0}=0.5 \mathrm{GeV} / \mathrm{fm}^{3}$ and $R_{0}=4.0 \mathrm{fm}$. The corresponding initial temperature and baryon density at $r=0$ are $T_{0}=137 \mathrm{MeV}$ and $n_{b 0}=0.36$ $\mathrm{fm}^{-3}$, respectively. They are obtained by using the state of equation of mixed ideal gas and $\epsilon_{0}$. The temperature $T_{0}=137 \mathrm{MeV}$ is lower than the estimated critical temperature, $T_{c}=160 \mathrm{MeV}$, of the transition between quark-gluon plasma and hadronic gas [9].

The two-particle Bose-Einstein correlation function is defined as the ratio of the two-particle momentum distribution $P\left(k_{1}, k_{2}\right)$ to the product of the single-particle momentum distribution $P\left(k_{1}\right) P\left(k_{2}\right)$. For an expanding source, $P(k)$ and $P\left(k_{1}, k_{2}\right)$ can be expressed as 7]

$$
\begin{aligned}
P(k) & =\int d^{4} x e^{-2 \mathcal{I} m \bar{\phi}_{s}(x)} \rho(x) A^{2}(\kappa(x), x), \\
P\left(k_{1}, k_{2}\right) & =\int d^{4} x_{1} d^{4} x_{2} e^{-2 \mathcal{I} m \bar{\phi}_{s}\left(x_{1}\right)} e^{-2 \mathcal{I} m \bar{\phi}_{s}\left(x_{2}\right)} \\
& \times \rho\left(x_{1}\right) \rho\left(x_{2}\right)\left|\Phi\left(\kappa_{1} \kappa_{2}: x_{1} x_{2} \rightarrow x_{d 1} x_{d 2}\right)\right|^{2},
\end{aligned}
$$

where $A(\kappa(x), x)$ is the magnitude of the amplitude for producing a pion with momentum $\kappa$ at $x$, which is proportional to the Bose-Einstein distribution characterized by the local temperature in the local frame of the cell at $x . \rho(x)$ is the pion-source density, which includes a primary source and the secondary source from $\Delta$ decay,

$$
\begin{aligned}
\rho(x) & =\rho^{\operatorname{prim}}(x)+\rho^{\text {second }}(x) \\
& =n_{\pi}(x) \delta(t)+\Gamma n_{\Delta}(x),
\end{aligned}
$$

where $\Gamma=120 \mathrm{MeV}$ is the width of $\Delta . e^{-2 \operatorname{Im} \bar{\phi}_{s}(x)}$ is the absorption factor due to multiple scattering [7, 11,

$$
e^{-2 \mathcal{I} m \bar{\phi}_{s}(x)}=\exp \left(-\int_{x}^{x_{f}} \sigma_{\mathrm{abs}}\left(\sqrt{s_{\pi N}}\right) n_{N}(x) d l\right),
$$

where $\sigma_{\text {abs }}\left(\sqrt{s_{\pi N}}\right)$ is the absorption cross section of $\pi+N \rightarrow \Delta$ at the center-of-mass energy $\sqrt{s_{\pi N}}$ and $d l$ is the spatial line element along the path of particle propagation. In Eq. (9), $\Phi\left(\kappa_{1} \kappa_{2}: x_{1} x_{2} \rightarrow x_{d 1} x_{d 2}\right)$ is the wave function for two-particles produced at $x_{1}$ and $x_{2}$ with momenta $\kappa_{1}\left(x_{1}\right)$ and $\kappa_{2}\left(x_{2}\right)$, and detected at $x_{d 1}$ or $x_{d 2}$ with momenta $k_{1}$ and $k_{2}$, respectively,

$$
\begin{aligned}
& \Phi\left(\kappa_{1} \kappa_{2}: x_{1} x_{2} \rightarrow x_{d 1} x_{d 2}\right) \\
& \quad=\frac{1}{\sqrt{2}}\left\{A\left(\kappa_{1}\left(x_{1}\right), x_{1}\right) A\left(\kappa_{2}\left(x_{2}\right) x_{2}\right)\right. \\
& \quad \times \exp \left[-i \int_{x_{1}}^{x_{f 1}} \kappa_{1}\left(x^{\prime}\right) \cdot d x^{\prime}-i k_{1} \cdot\left(x_{d 1}-x_{f 1}\right)\right] \\
& \quad \times \exp \left[-i \int_{x_{2}}^{x_{f 2}} \kappa_{2}\left(x^{\prime}\right) \cdot d x^{\prime}-i k_{2} \cdot\left(x_{d 2}-x_{f 2}\right)\right] \\
& \quad+A\left(\kappa_{1}\left(x_{2}\right), x_{2}\right) A\left(\kappa_{2}\left(x_{1}\right), x_{1}\right) \\
& \quad \times \exp \left[-i \int_{x_{2}}^{x_{f 2}^{\prime}} \kappa_{1}\left(x^{\prime}\right) \cdot d x^{\prime}-i k_{1} \cdot\left(x_{d 1}-x_{f 2}^{\prime}\right)\right] \\
& \left.\quad \times \exp \left[-i \int_{x_{1}}^{x_{f 1}^{\prime}} \kappa_{2}\left(x^{\prime}\right) \cdot d x^{\prime}-i k_{2} \cdot\left(x_{d 2}-x_{f 1}^{\prime}\right)\right]\right\},
\end{aligned}
$$

where $x_{f 1}$ and $x_{f 2}$ are the freeze-out points corresponding to the particles 1 and 2 produced at $x_{1}$ and $x_{2}$ and detected at $x_{d 1}$ and $x_{d 2}$, respectively. $x_{f 1}^{\prime}$ and $x_{f 2}^{\prime}$ are the freeze-out points corresponding to the particles 1 and 2 produced at $x_{1}$ and $x_{2}$ and detected at $x_{d 2}$ and $x_{d 1}$, respectively. As we consider mainly two particles whose momenta are nearly parallel, we approximately have $x_{f 1}^{\prime}=x_{f 1}$ and $x_{f 2}^{\prime}=x_{f 2}$ [7, 12]. From Eqs. (8), (9), and (12) the correlation function $C\left(k_{1}, k_{2}\right)=$ $P\left(k_{1}, k_{2}\right) / P\left(k_{1}\right) P\left(k_{2}\right)$ can be written as [7]

$$
\begin{aligned}
C\left(k_{1}, k_{2}\right)= & 1+\mid \int d^{4} x e^{i\left(k_{1}-k_{2}\right) \cdot x+i \phi_{c}\left(x, k_{1} k_{2}\right)} \\
& \times\left. e^{-2 \operatorname{Im} \bar{\phi}_{s}(x)} \rho_{\mathrm{eff}}\left(x ; k_{1} k_{2}\right)\right|^{2},
\end{aligned}
$$

where $\rho_{\text {eff }}$ is the effective density

$$
\begin{gathered}
\rho_{\text {eff }}\left(x ; k_{1} k_{2}\right)=\frac{\sqrt{f_{\text {init }}\left(\kappa_{1}(x), x\right)} \sqrt{f_{\text {init }}\left(\kappa_{2}(x), x\right)}}{\sqrt{P\left(k_{1}\right) P\left(k_{2}\right)}}, \\
f_{\text {init }}(\kappa(x), x)=\rho(x) A^{2}(\kappa(x), x)
\end{gathered}
$$




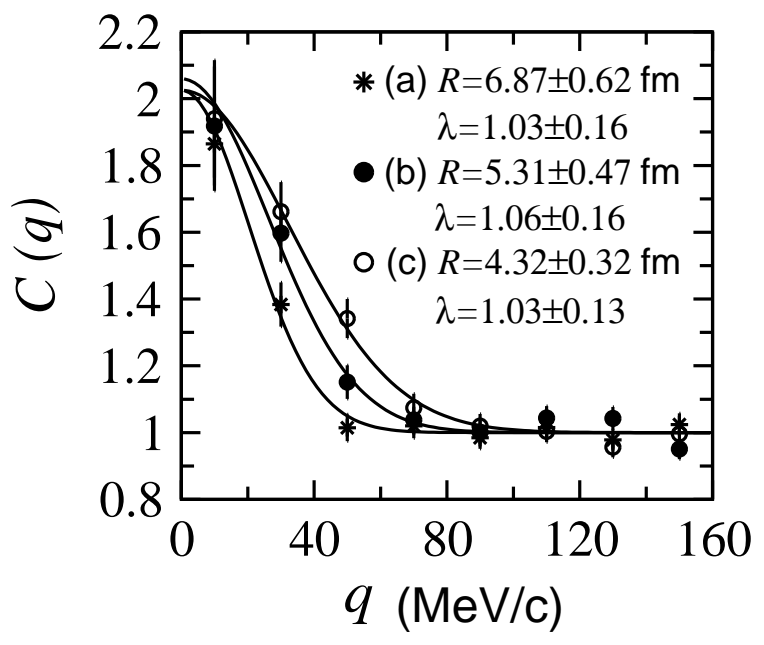

FIG. 2: Two-pion correlation functions $C(q)$ for the cases (a) freeze-out, (b) present formulation including absorption of pions by nucleons, and (c) without absorption.

is the phase space distribution of particle sources, and

$\phi_{c}\left(x, k_{1} k_{2}\right)=-\int_{x}^{x_{f}}\left\{\left[\kappa_{1}\left(x^{\prime}\right)-\kappa_{2}\left(x^{\prime}\right)\right]-\left[k_{1}-k_{2}\right]\right\} \cdot d x^{\prime}$.

It can be seen that the two-pion correlation function is related to the phase space distribution of the pion production source, modified by an absorption factor arising from multiple scattering and a phase factor $\phi_{c}$ from the collective expansion.

Knowing the hydrodynamical solution as the spacetime variations of density, velocity, and thermodynamical functions of the fluid cells, we can construct the trajectories of a pair of test pions which start initially at $x_{1}$ and $x_{2}$ and emerge finally with momenta $k_{1}$ and $k_{2}$ at freeze-out. The knowledge of the space-time trajectories of the pair of pions allows one to calculate the path integrals in the wave function $\Phi$ of Eq. (12) and the function $P\left(k_{1}, k_{2}\right)$ and $P(k)$ after summing over all source elements in Eqs. (8) and (9). The correlation function $C(q)$ of the relative momentum of the two particles, $q=\left|\mathbf{k}_{\mathbf{1}}-\mathbf{k}_{\mathbf{2}}\right|$, can be constructed from $P\left(k_{1}, k_{2}\right)$ and $P\left(k_{1}\right) P\left(k_{2}\right)$ by integrating over the average momentum $\left(\mathbf{k}_{\mathbf{1}}+\mathbf{k}_{\mathbf{2}}\right) / 2$ [13]. The HBT radius $R$ can then be extracted by parameterizing the correlation function $C(q)$ as

$$
C(q)=1+\lambda e^{-q^{2} R^{2}} .
$$

In our model calculation, we shall consider the freezeout configuration to be characterized by a freeze-out temperature of $T_{f}=0.5 T_{0}$. We calculate first the correlation function $C(q)$ for the case (a) when the detected pions are assumed to originate only from the freeze-out configuration. This is the configuration considered in the conventional description of intensity interferometry with collective expansion where one assumes a chaotic source at freeze-out due to random pion rescattering.

We calculate next the case (b) using the present formalism to take into account the effects of multiple scattering and collective expansion with quantum probability amplitudes in a path-integral framework. We use the experimental absorption cross section [14] represented by the model of the absorption of a pion by a nucleon forming a delta resonance. For further comparison, we calculate $C(q)$ for the case (c) in which the detected pions originate from the source under the hydrodynamical expansion with no absorption.

Figure 2 shows results of the two-pion interferometry for these cases. The conventional freeze-out configuration [case (a)] leads to an extracted HBT radius of $6.87 \mathrm{fm}$. When there is no absorption of pions [case (c)], the HBT radius of $4.32 \mathrm{fm}$ is small. The HBT radius obtained by using the present formalism [case (b)] leads to an HBT radius of $5.31 \mathrm{fm}$ which lies between the radii of case (a) and case (c). The extracted HBT radius depend on the description of the pion source and absorption.

We conclude that for a pion source of the type considered, the HBT radius extracted from the freeze-out configuration is substantially greater than the radius obtained when effects of multiple scattering and collective expansion on the HBT interferometry have been properly taken into account using quantum probability amplitudes in a path-integral formalism. As the latter description is a more realistic description of the HBT interferometry, the conventional assumption that the HBT interferometry measures the distribution of the freeze-out configuration is therefore subject to question.

We have carried out model calculations for some idealized configurations in order to illustrate the main features of the effects under consideration. These features will remain the same for more realistic space-time configurations. In this paper the hadronic gas is taken to be an ideal gas. For a more realistic case, one should consider the volume correction [15, 16], which will lead to a slightly lower temperature and lower density of hadronic gas but will hardly affect the main features of the HBT interferometry considered here. The freeze-out temperature $T_{f}=0.5 T_{0}$ considered in this paper is lower than the freeze-out temperatures $T_{f}=0.7 T_{c}$ and $0.9 T_{c}\left(T_{c}=160\right.$ $\mathrm{MeV}$ ) considered for a zero net baryon density case [9] because the cross section between pion and baryon is greater than that between pions. The HBT radii of the three cases considered in this paper will increase when the freeze-out temperature decreases but the main feature concerning the relative magnitudes of the HBT radii is unaltered.

WNZ would like to thank Drs. T. Barnes, V. Cianciolo, and G. Young for their kind hospitality at Oak Ridge National Laboratory. This research was supported by the National Natural Science Foundation of China under Contract No.10275015 and by the Division of Nuclear Physics, US DOE, under Contract No. DE-AC0500OR22725 managed by UT-Battle, LC. 
[1] For a review see Chapter 17 of C. Y. Wong, Introduction to High-Energy Heavy-Ion Collisions, World Scientific Publishing Company, 1994; U. A. Wiedemann, U. Heinz, Phys. Rept. 319, 145 (1999); R. M. Weiner, Phys. Rept. 327, 249 (2002).

[2] K. Adcox et al., Phys. Rev. Lett. 88, 192302 (2002).

[3] C. Adler et al., Phys. Rev. Lett. 87, 082301 (2001).

[4] D. Molnár and M. Gyulassy, Phys. Rev. Lett. 92, 052301 (2004).

[5] S. Pratt, Nucl. Phys. A 715, 389c (2003).

[6] U. Heinz and P. Kolb, Nucl. Phys. A 702, 269 (2002).

[7] Cheuk-Yin Wong, J. Phys. G 29, 2151 (2003).

[8] P. Kolb and U. Heinz, nucl-th/0305084

[9] D. H. Rischke, M. Gyulassy, Nucl. Phys A 608, 479
(1996); D. H. Rischke, nucl-th/9809044

[10] H. Miao, Z. Ma, C. Gao, Commun. Theor. Phys. 38, 698 (2002).

[11] Cheuk-Yin Wong, and R. Glauber, to be submitted.

[12] Cheuk-Yin Wong, J. Phys. G 30, S1053 (2004).

[13] W. N. Zhang, Y. M. Liu, S. Wang, Q. J. Liu, J. Jiang, D. Keane, Y. Shao, S. Y. Chu, and S. Y. Fung, Phys. Rev. C 47, 795 (1993).

[14] K. Hagiwara et al., Phys. Rev. D 66, 010001 (2002).

[15] S. P. Baranov and L. V. Fil'kov, Z. Phys. C 44, 227 (1989).

[16] W. N. Zhang, G. X. Tang, X. J. Chen, L. Huo, Y. M. Liu, and S. Zhang, Phys. Rev. C 62, 044903 (2000). 